\title{
Improved chemistry restraints for crystallographic refinement by integrating Amber molecular mechanics in Phenix
}

\author{
David A. Case, Pawel A. Janowski, Nigel W. Moriarty, Jason M. Swails, and Paul D. Adams \\ Rutgers University, Dept. of Chemistry and Chemical Biology, Piscataway, NJ 08854 \\ Molecular Biophysics \& Integrated Bioimaging Division, Lawrence Berkeley National \\ Laboratory, Berkeley, CA 94720 \\ University of California at Berkeley, Dept. of Bioengineering, Berkeley, CA 94720
}

\begin{abstract}
The refinement of biomolecular crystallographic models relies on the use of geometric restraints to help address the paucity of experimental data typically observed in these experiments. Inaccuracies in these restraints can lead to errors in the resulting models. Here we present the integration of the Amber molecular dynamics software with Phenix crystallographic refinement, which makes available the full all-atom Amber molecular mechanics force field for more accurate modelling of biomolecular chemistry. The advantages of the Amber force field include a carefully derived set of torsion angle potentials, an extensive and flexible set of atom types, Lennard-Jones treatment of non-bonded interactions and a full treatment of crystalline electrostatics. The new combined method was tested against conventional geometry restraints for over six thousand protein and nucleic acid structures. Structures refined with the new method show improved model quality. In particular, clash scores are improved and most structures display a better MolProbity score. Additionally, the modelling of electrostatics leads to structures that exhibit on average more hydrogen bonds than those refined with traditional geometry restraints. While R-free remains essentially unchanged, most models possess a smaller $R_{\text {work }}-R_{\text {free }}$ gap, indicating less overfitting to the experimental data. We observe that model improvements are greatest at lower resolutions and with poorer starting models. This work opens the door to the future development of more advanced applications such as Amber-based ensemble refinement, quantum mechanical representation of active sites, and improved geometric restraints for simulated annealing or realspace refinement.
\end{abstract}

